\title{
Situación laboral y calidad de vida de las personas con discapacidad física Huancayo
}

\section{Lulay kaynin nunakunap allin kawsaynin mana allisha nunakayniyu Wankayu}

\author{
Ikantokotaiga ira matsigengapage iramasitapage kara \\ timagantsikë Huancayo (katsingagiterikë) intaigi irantanepage
}

\section{Okantakoyetari yantabeyeti aisati isabikayeti amashiyetachari Huancayoki}

\author{
Recibido: 7 abril 2019 Corregido: 20 agosto 2019 Aprobado: 28 octubre 2019
}

\begin{abstract}
Eugenia Fabián Arias, Nacionalidad: Peruana Filiación: Universidad Nacional del Centro del Perú; Correo: eugefabian@uncp.edu.pe; ORCID: https://orcid.org/oooo-0002-7587-1279

Luz María, Vilcas Baldeón, Nacionalidad: Peruana Filiación: Universidad Nacional del Centro del Perú, ORCID: https://orcid.org/0000-0003-1165-7246
\end{abstract}

Yovana Luiza, Alberto Bueno, Nacionalidad: Peruana Filiación: Universidad Nacional del Centro del Perú, Correo: yalberto@uncp.edu.pe ORCID: https://orcid.org/0000-0003-1207-9273

\section{Resumen}

La situación laboral es la condición que se encuentra las PcDF y la calidad de vida es la satisfacción experimentada por su condición de vida. El objetivo del estudio fue conocer la situación laboral y calidad de vida de las personas con discapacidad física de 18 a 65 años del OMAPED-Huancayo. El tipo de estudio fue básico, nivel descriptivo, carácter cuantitativo, con una muestra de 280 PcDF; el método fue de análisis síntesis, técnica encuesta, cuestionario escala; válido y confiable o,7 por el coeficiente Alfa de Crombach. Resultados: Del $68,9 \%$ de las PcDF del sector informal sus ingresos económicos son irrisorios y $31,1 \%$ del sector formal son igual o mayor a la remuneración mínima, siendo la calidad de vida regular en todas sus dimensiones $(2,1624)$. En conclusión, la situación laboral de las PcDF, del sector formal e informal es diferenciada por sus ingresos y la calidad de vida es regular.

\section{Palabras clave:}

Situación laboral, calidad de vida.

\section{Lisichiku limaykuna:}

Lulay kaynin, allin kawsay.

Nibarintsipage Katingaro: Ikantaiga antagansipagekë aike kanina inimaige.

\section{Ñantsipe ayoyeteri:}

Okantakoyetari yantabeyeti, okantayetari isabikayeti. 


\title{
Work Situation and Quality of Life of People with Physical Disabilities Huancayo
}

\begin{abstract}
The employment situation is the condition found in the PCDF and the quality of life is the satisfaction experienced by their condition of life. The objective of the study was to know the employment situation and quality of life of people with physical disabilities between 18 and 65 years of age of OMAPED-Huancayo. The type of study was basic, descriptive level, quantitative character, with a sample of 280 PcDF; The method was synthesis analysis, survey technique, questionnaire scale; valid and reliable 0.7 for Crombach's alpha coefficient. Results: Of the $68.9 \%$ of the informal sector, PcDF their economic income is derisory and $31.1 \%$ of the formal sector are equal to or greater than the minimum remuneration, being the quality of life regular in all its dimensions $(2,624)$. In conclusion, the employment situation of the PcDF, of the formal and informal sector is differentiated by their income and the quality of life is regular.
\end{abstract}

\section{Situação laboral e qualidade de vida das pessoas com incapacidade/deficiência física (PCDF) de Huancayo}

\section{Resumo}

A situação laboral é a condição que se encontra nas PCDF e a qualidade de vida é a satisfação experimentada por sua condição de vida. O objetivo do estudo foi conhecer a situação laboral e a qualidade de vida de pessoas com incapacidade/deficiência física entre 18 e 65 anos de idade do OMAPED-Huancayo. O tipo de estudo foi básico, nível descritivo, caráter quantitativo, com amostra de 280 PCDF; O método foi análise de síntese, técnica de enquete, escala de questionário; o,7 válido e confiável para o coeficiente alfa de Crombach. Resultados: dos 68,9\% do setor informal PCDF, a renda econômica é irrisória e 31,1\% do setor formal é igual ou superior à remuneração mínima, sendo a qualidade de vida regular em todas as suas dimensões $(2,1624)$. Em conclusão, a situação de emprego do PCDF, do setor formal e informal, é diferenciada por sua receita e a qualidade de vida é regular.

\section{Keywords}

Work situation, quality of life.

\section{Palavras-chave:}

Situação laboral, qualidade de vida.

\section{Datos de los autoras}

Eugenia Fabián Arias: Investigador y docente en investigación. Doctor en Ciencias de la Educación. Magister en didáctica universitaria.

Luz María Vilcas Baldeón: Investigador y docente en diagnóstico social. Magister en Educación-Mención en Enseñanza Estratégica.

Yovana Luiza Alberto Bueno: Investigador y docente en proyectos sociales. Magister en administración. 


\section{Introducción}

La organización internacional del trabajo según las estadísticas disponibles, evidencia que las personas con discapacidad tienen menos probabilidades que las personas que no padecen ninguna discapacidad con respecto a la empleabilidad en el mercado de trabajo activo y cuando las personas con discapacidad consiguen un trabajo, es más probable que se trate de un empleo poco remunerado con pocas perspectivas y malas condiciones de trabajo. (OIT; 2012:3).

En el mundo del trabajo competitivo en las empresas normalizadas, las personas con discapacidad, a menudo son excluidas por patrones de discriminación, generando altas tasas de desempleo en este colectivo. Mercado (2013) citado por Vidal y Cornejo (2016) "señala que la intensidad de la discapacidad constituye un elemento de especial trascendencia condicional de la implicación en el mercado laboral”.

La integración laboral de las personas con discapacidad es uno de los ámbitos de actuación relacionados con la discapacidad de acuerdo a la Organización Mundial de la Salud (OMS) y al Banco Mundial (2011:7) "más de mil millones de personas viven con algún tipo de discapacidad, ósea al rededor del $15 \%$ de la población mundial”.

El modelo de calidad de vida de Schalock y Verdugo (2002), es integrada por las siguientes dimensiones; bienestar emocional, relaciones interpersonales, bienestar material, desarrollo personal, bienestar físico, autodeterminación inclusión social y derechos, ha sido validado por números estudios internacionales (Jenaro et al., 2005; Schalock et al., 2005), citado por Larrayos (2014). La Calidad de vida es equivalente a la suma de los puntajes de la condición de vida objetivamente medibles en una persona, tales como salud física, condiciones de vida, relaciones sociales, actividades funcionales u ocupación. Este tipo de definición permitirá comparar a una persona con otra desde indicadores estrictamente objetivos, sin embargo, estos, parecieran que apuntan más una cantidad que calidad de vida.

La calidad de vida de las personas, en su más amplio sentido, resguardado a través de la declaración de los derechos humanos, es el quehacer fundamental del Trabajo Social. Las personas en situación de discapacidad tienen los peores niveles de calidad de vida, esto se debe principalmente a la falta de oportunidades al acceso a la salud, educación y empleo. Es así que la perspectiva social de la discapacidad no ha evolucionado lo suficiente como para orientar las políticas sociales y descartar las discriminaciones que sigue sufriendo el colectivo. (Ávila; 2011)

En el caso de las políticas de discapacidad se trata de mostrar que estas no podrán alcanzar sus objetivos y los impactos deseados a menos que se entienda que las desventajas que afectan a las personas con discapacidad no se encuentran tanto en sus deficiencias personales sino más bien y principalmente en las barreras físicas y mentales (actitudes, prejuicios, discriminación) que la sociedad y el Estado construyen.

La situación laboral en la que se encuentran PcDF del OMAPED Huancayo, es una gran preocupación en la actualidad, el 11\% de la población corresponde a las personas con discapacidad, quienes se encuentran en una situación de vulnerabilidad y exclusión social, que en su mayoría de los casos no tienen oportunidad laboral, en ese sentido el objetivo de la investigación fue conocer la situación laboral y calidad de vida de las personas con discapacidad física del OMAPED - Huancayo. 


\section{Situación laboral de las personas con discapacidad física}

\section{Las características de las PcDF}

El 71.4\% de las personas con discapacidad física tienen de 40 a 59 años de edad; el $62.1 \%$ son de género masculino; mayormente el $41.9 \%$ residen en el distrito de el Tambo, $35.4 \%$ en el distrito de Huancayo y el $\mathbf{2 2 . 9 \%}$ viven en el distrito de Chilca; su estado civil es $53 \%$, casados, $35 \%$ convivientes y solteros $13 \%$.

\begin{tabular}{|c|c|c|c|c|c|}
\hline \multicolumn{6}{|c|}{$\begin{array}{c}\text { Tabla } \mathrm{N}^{\circ} \mathbf{1} \\
\text { Grado de instrucción }\end{array}$} \\
\hline & & Frecuencia & Porcentaje & $\begin{array}{l}\text { Porcentaje } \\
\text { válido }\end{array}$ & $\begin{array}{l}\text { Porcentaje } \\
\text { acumulado }\end{array}$ \\
\hline \multirow[t]{5}{*}{ Válido } & Sin Estudios & 12 & 4,3 & 4,3 & 4,3 \\
\hline & $\begin{array}{l}\text { Primaria completa/ } \\
\text { incompleta }\end{array}$ & 45 & 16,1 & 16,1 & 20,4 \\
\hline & $\begin{array}{l}\text { Secundaria completa/ } \\
\text { incompleta }\end{array}$ & 117 & 41,8 & 41,8 & 62,1 \\
\hline & Técnico/superior & 106 & 37,9 & 37,9 & 100,0 \\
\hline & Total & 280 & 100,0 & 100,0 & \\
\hline
\end{tabular}

En la tabla $\mathrm{N}^{\circ} 1$ se percibe que $41.8 \%$ tienen estudios de secundaria completa e incompleta, el $37.9 \%$ tienen especialidades técnicas o superiores; $16.1 \%$ tienen primaria completa e incompleta, y $4.3 \%$ no estudió; de allí que el $68.9 \%$ de las PcDF que trabajan tienen empleo informal y/o comercio ambulatorio. Sólo el 31.1\% de las PCDF tienen un empleo formal; laborando en diversas instituciones públicas o privadas de nuestra localidad. Estos datos se evidencian en Cardona, Agudelo, Restrepo, Sánchez, y Segura (2014, p.8) cuando concluyen "que las personas que indicaron tener una mala o regular calidad de vida, solo alcanzaron estudios primarios". Asimismo Inquilla (2014) sostiene que “...las personas con discapacidad presentan dificultades para acceder a la educación y al mercado laboral se ponen con frecuencia en situación de vulnerabilidad”. (p.1).

\begin{tabular}{|c|c|c|c|c|c|}
\hline \multicolumn{6}{|c|}{$\begin{array}{c}\text { Tabla } \mathrm{N}^{\circ} \mathbf{2} \\
\text { Mercado Laboral }\end{array}$} \\
\hline & & Frecuencia & Porcentaje & $\begin{array}{c}\text { Porcentaje } \\
\text { válido }\end{array}$ & $\begin{array}{l}\text { Porcentaje } \\
\text { acumulado }\end{array}$ \\
\hline \multirow[t]{3}{*}{ Válido } & Empleo informal & 193 & 68,9 & 68,9 & 68,9 \\
\hline & Empleo formal & 87 & 31,1 & 31,1 & 100,0 \\
\hline & Total & 280 & 100,0 & 100,0 & \\
\hline
\end{tabular}

En la tabla $\mathrm{N}^{\circ} 2$, observamos que el empleo de las PCDF de las OMAPEDS Huancayo, es predominantemente informal 68.9\%(193), seguido del formal 31.1\%(87) quienes vienen laborando en diversas instituciones públicas y privadas, estos datos se sostienen en Bara (2015) cuando indica que las personas con discapacidad física crónica participan menos en el trabajo remunerado y voluntario y están menos satisfechos con sus vidas que las personas sin problemas de salud .Es decir que las PcDF, no tiene acceso al trabajo remunerado en instituciones públicas y privadas, debido a que la mayoría tienen un bajo nivel de instrucción ; por lo que no acceden a participar en los concursos públicos por méritos para ocupar una plaza, deduciendo que care- 
cen de una calificación, por su bajo nivel de instrucción ,que restringe su posibilidad de acceder a un empleo formal.

Respecto a los cargos o responsabilidades en el centro de trabajo de las PcDF, el 8.6\%(24) son jefes o responsables del área donde trabajan, $12.14 \%(34)$ trabajan como asistentes, el $12.86 \%(34)$ son profesores.

$52.9 \%(148)$ de la PCDF se dedican a la informalidad, tienen su propio negocio, estos resultados se sustentan en Maldonado (2004) cuando mencionan, que la tasa de desempleo no es la mejor caracterizadora de la situación de exclusión laboral, que padecen las personas con discapacidad, a pesar de que ésta es casi el doble de la tasa de desempleo que experimentan las personas sin discapacidad. Efectivamente las PcDF, presentan mayor desempleo porque no tiene una oportunidad laboral al igual que los demás pobladores del país y para subsistir se dedican al trabajo informal de donde obtienen ingresos económicos irrisorios. Este problema es debido a múltiples dificultades que tiene para acceder a la educación. Además, se puede señalar que la mayoría no tiene perspectivas de empleo porque muchos de los obstáculos que enfrentan en su búsqueda o en el lugar que trabajan se deben no solo a las barreras sociales, sino también a su propia discapacidad.

Por otra parte, Kaye (2009) enunciado en Alcover y Pérez (2011) manifiestan que las personas con discapacidad se encuentran relegadas de una manera desproporcionada a un acceso al mercado de trabajo, es frecuente el subempleo al que se ven obligadas las personas con discapacidad quienes perciben bajos salarios y una menor seguridad y estabilidad laboral.

Sin embargo, de manera general las personas con discapacidad siguen experimentado formas comunes de discriminación como un alto nivel de desempleo o incluso la exclusión del mercado laboral

\section{Tabla $\mathbf{N}^{\circ} 3$ Ingresos económicos}

\begin{tabular}{ccc|c|c|c} 
& Frecuencia & Porcentaje & $\begin{array}{c}\text { Porcentaje } \\
\text { válido }\end{array}$ & $\begin{array}{c}\text { Porcentaje } \\
\text { acumulado }\end{array}$ \\
\hline \multirow{3}{*}{ Válido } & Menos de $\mathrm{S} / 300$ & 145 & 51.8 & 51.8 & 51.8 \\
\cline { 2 - 4 } & $\mathrm{S} / 301-\mathrm{S} / 700$ & 62 & 22,1 & 22,1 & 73,9 \\
\cline { 2 - 5 } & $\mathrm{S} / 850$ a más & 73 & 26,1 & 26,1 & 100,0 \\
\cline { 2 - 5 } & Total & 280 & 100,0 & 100,0 & \\
\hline
\end{tabular}

En la tabla $\mathrm{N}^{\circ} 3$, observamos que los ingresos económicos de las PcDF, son irrisorios, porque el $51.8 \%$ percibe menos de $\mathrm{S} / 300$, seguido por el $22.1 \%$, que percibe de $\mathrm{S} / 301$ a $\mathrm{S} / 700$ mensuales; y $26.1 \%$ declaran ganar más de $S / 850$, estarían aquellos que se encuentran en el empleo formal, estos resultados se sustentan en Meyer y Mok (2018) cuando manifiestan que la discapacidad está asociada con resultados económicos pobres básicamente de ingresos económicos. Se evidencia fundamentalmente en aquellos que se dedican al empleo informal como es la venta de dulces, frutas, comida y otros.

De la totalidad de los PcDF que se encuentran en la informalidad, manifestaron que $46.48 \%$ "nunca" cuentan con mobiliario para la venta de sus productos, por falta de dinero, el $31.92 \%$ 
"siempre" cuenta con mobiliario, así como carretas y vitrinas improvisadas y el $21.60 \%$ considera que solo "a veces", cuentan con mobiliario para trabajar cuando les facilitan otras personas.

Por otra parte , el 69.48\% PcDF respondieron que "nunca" cuentan con permiso de la municipalidad para realizar su negocio ,porque son actividades ambulatorias y están abocados solo al comercio de poca inversión, como la venta de dulces y frutas picadas en las calles y/o alrededores de los mercados; el 22.54\% respondieron que "siempre" cuentan con permiso de la municipalidad, porque las actividades que realizan como la venta de periódicos en quioscos, pequeños restaurants, talleres de duplicado de llaves, etc. requieren de permiso de la municipalidad y el 7.98\% respondieron que "a veces", solicitan permiso de la municipalidad en fechas festivas como semana santa, día de la madre y navidad.

El 31.1\%, de los PcDF ,que tienen un empleo formal en diversas instituciones públicas o privadas de nuestra localidad, reciben los beneficios de acuerdo a las normas laborales vigentes; como el seguro social, aguinaldos, CTS, indemnización, subsidios y descanso médico. Los cargos o responsabilidades que desempeñan se distribuyen en; $12 \%$ como ayudante o asistente, $12 \%$ docentes o profesores y el $8 \%$ son jefes o responsables de áreas.

\section{Tabla $N^{\circ} 4$ \\ Dificultades para su desplazamiento al trabajo}

\begin{tabular}{ccc|c|c|c} 
& & Frecuencia & Porcentaje & Porcentaje válido & $\begin{array}{c}\text { Porcentaje } \\
\text { acumulado }\end{array}$ \\
\hline \multirow{3}{*}{ Válido } & Siempre & 67 & 23,9 & 23,9 & 23.9 \\
\cline { 2 - 4 } \cline { 5 - 6 } & A veces & 151 & 53.9 & 53.9 & 77,2 \\
\cline { 2 - 6 } & Nunca & 62 & 22,2 & 22,2 & 100,0 \\
\cline { 2 - 6 } & Total & 280 & 100.0 & 100.0 & \\
\hline
\end{tabular}

En la tabla $\mathrm{N}^{\circ}$ 4, visualizamos que el $53.9 \%$ de las PcDF tienen dificultades para su desplazamiento, porque algunas veces los medios de transporte no les trasladan, por su silla de ruedas, el $23.9 \%$ "siempre" tiene dificultades porque necesita el apoyo de otra persona y el $22.2 \%$ manifiestan que "nunca" tienen dificultades porque sus limitaciones no son tan severas y para desplazarse no requieren de ayuda de otras personas o familiares como se presentan en los otros casos.

\section{Calidad de vida}

En la dimensión de relaciones interpersonales es buena porque el $48.2 \%$ (135) considera que "siempre" tienen facilidades para relacionarse con otras personas y referente a la familia el 63\% (178) siente tener buena relación. Asimismo el 60.4\% (169) manifiestan que la relación con sus amigos y compañeros de trabajo es buena y el $38.9 \%$ (109) siempre tienen amigos de su entera confianza; y además el $42.9 \%$ (120) siempre reciben apoyo de sus familiares. Estos resultados se sustentan en Morales, Gaviria, y Cuadrado (2007) cuando definen las relaciones interpersonales como aquellas que incluyen las relaciones de amistad, pareja, entre padres e hijos, entre compañeros y otros por el estilo, forman parte esencial de la vida social, sin ellos los humanos no podrían sobrevivir en el más estricto sentido biológico. Es decir, las personas con discapacidad física tienen buenas relaciones con sus familiares y amistades.

En la dimensión del bienestar material, la calidad de vida, es mala, porque el 59.6\% (167) no reciben ningún tipo de apoyo de instituciones, ni organizaciones de ninguna índole, así mismo el $61.4 \%$ (172) no se encuentran en la capacidad de ahorrar para el futuro por los ingresos eco- 
nómicos precarios que tienen. Asimismo, el 66.4\% (186) manifestaron que nunca tienen una cuenta bancaria debido a que no tienen capacidad de ahorro. Sin embargo, tienen viviendas con los servicios básicos $67.1 \%$ (188), esto es porque la mayoría de ellos, solo tienen casa de material rustico, y se sienten cómodos y satisfechos 38.9\% (109) porque tienen un espacio donde vivir. Estos resultados se sostienen en Velarde y Ávila (2002, p.349) cuando manifiesta "que la pobreza deriva en falta de servicios, en desnutrición y en un mayor riesgo de adquirir enfermedades transmisibles".

La calidad de vida de las PCDF de 18 a 65 años del OMAPED Huancayo en la dimensión de bienestar emocional, es regular, porque tienen una media de 1.8929. Asimismo el 50.4\%(141), responden que "a veces" se sienten alegres y contentos pese a las diversas necesidades que tienen. También el 41.8\%(117) se consideran "a veces" personas exitosas o talentosas, porque pese a su discapacidad consideran que tienen muchas habilidades para salir adelante. Además, el 71.1\% (199) "a veces" se sienten preocupados o estresados por los ingresos precarios por el trabajo informal que realizan.

Estos resultados, se sustentan en Álvarez (2014) cuando, declara que las personas con discapacidad intelectual (PCDF) tienen una gran desventaja respecto al resto de la población, ya que dependen para lograr la plena integración social, no solo de sus competencias y características personales, sino del apoyo que les facilita el entorno. Es decir aquellos que tienen acceso al trabajo formal, presentan una mejor calidad de vida en relación al bienestar emocional.

En la dimensión de desarrollo personal es regular con una media de 2,077, por que el 53.6\%, carecen de carrera profesional y el 30\% respondieron que "siempre" han ejercido su carrera de técnico o profesional, en este rubro se encuentra las personas del sector formal.

El 50.7\%, siempre hacen uso de sus habilidades sociales para las actividades que desarroIlan y el $55.7 \%$ a veces participan en actividades de desarrollo personal como capacitaciones. Estos datos se sustentan en Cardona, Agudelo, Restrepo, y Segura (2012) cuando evidencian una relación directamente proporcional entre la edad y la presencia de algún tipo de discapacidad; adicional a esto existen factores económicos, educativos y de salud que deterioran su calidad de vida y aumentan su vulnerabilidad.

Por otra parte Bolaños (2011), sostiene que el desempeño de las personas con discapacidad en el ámbito laboral , cobra un realce superlativo cuando nos damos cuenta que este grupo, en situación de vulnerabilidad ,necesita también satisfacer necesidades propias de desarrollo y crecimiento personal, en efecto es a través de un trabajo que las personas con discapacidad pueden volverse realmente independientes y en consecuencia ser asedor de su propio destino, también se respalda en el modelo de (Borthwick- Duffy, 1992; Felce \& Perry 1995) citado por Urzúa y Caqueo (2012;p.3) cuando señalan que la calidad de vida es definida como la satisfacción experimentada por una persona con dichas condiciones de vida, la calidad de vida es sinónimo de la satisfacción personal, existen estándares relacionados con las condiciones de vida, pero la satisfacción que expresa la persona con cada uno de estos y la combinación de los mismos genera un sentimiento de satisfacción general con la propia vida y lo que en definitiva define la calidad de vida.

La calidad de vida de las PCDF de 18 a 65 años del OMAPED Huancayo en la dimensión de Bienestar físico: Referente a la atención sanitaria que reciben, es regular porque tienen una media de 2.1665 con tendencia abajo y/o malo. Porque el 54\% (152) siempre reciben atención sanitaria en el SIS y el 37.1\% (119) en ESSALUD y el 8.6\% refieren que solo "a veces" reciben 
atención médico sanitario, deduciendo que estas personas son atendidas por medicinas caseras y de forma personal. Además en cuanto a sus enfermedades y dolencia a parte de su discapacidad el $48.6 \%$ respondieron que "a veces" sufren de dolencias y el $25.4 \%$ "siempre" sufren de enfermedades o dolencias; en cuanto a la pertenencia en alguna liga deportiva o equipo, el 75.4\% "nunca" han pertenecido a una organización deportiva; estos resultados se sustentan en Brown, Bayer y Mac Farlanes (1992) "se da en la discrepancia de los logros de una persona y sus necesidades" (p.2). El modelo propuesto consiste en una combinación de medidas objetivas relacionadas con los ingresos entorno, salud, crecimiento personal y adquisición de habilidades y de medidas subjetivas que tienen que ver con la satisfacción por la vida en general.

En la dimensión de autodeterminación es regular (con tendencia a baja) por que el 53.6\% (150) señalaron que nunca participan en ninguna organización religiosa, cultural o artística; el $42.5 \%$ (119) manifiestan que solo a veces participan en eventos sociales; $42.1 \%$ (118) declararon que en situaciones difíciles controlan sus impulsos y emociones, el $58.9 \%$ (165) a veces no logran sus metas y el $56.4 \%$ (158) siempre practican valores con frecuencia. Estos datos se sustentan en Hernández (2004) cuando explica que para eliminar las barreras que dificultan la autonomía de las personas con discapacidad, se debe integrarlos en todo el nivel, en procesos de reflexión y toma de decisiones a nivel político, social y económico, de planeación y recreativo, favoreciendo la participación en actividades específicas, (p.5)

\section{Desmitificando la incapacidad y eliminando el sensacionalismo y las exageraciones en este rubro.}

Dimensión de inclusión social, referente a la participación en espacios de toma de decisiones es regular con una media de 2, 613 con tendencia a baja (es mala) porque el 31.1\% manifestaron que "nunca" participan en espacios de toma de decisiones y el $30 \%$ respondieron que "a veces" en espacios de toma de decisiones. El 48.2\% (135) nunca ha sido elegido como representante de alguna organización y el 63.6\% (178) nunca participan en programas sociales, organizaciones o instituciones de la región y el 70\% (196) no desempeñan roles ni funciones en la comunidad; asimismo el $73.6 \%$ (206) nunca han tenido apoyo social del gobierno regional, local e institucional , estos resultados se respaldan en Maldonado (2004) cuando menciona que la variable adecuada para aproximarnos a la extensión de la exclusión es la tasa de participación, la misma que apenas es de $24 \%$ hacia el 2003 para el caso de Lima Metropolitana. Ello contrasta con el $64 \%$ de personas con discapacidad que se encuentran participando en el mercado laboral hacia el mismo periodo", por otra parte, Larrayoz (2014) menciona “...que, en nuestra sociedad, el empleo constituye el elemento clave para la integración social de la persona con discapacidad, en este sentido, tienen una gran desventaja respecto al resto de la población, ya que dependen, para lograr la plena integración social, no solo de sus competencias y características personales, sino de los apoyos que les proporcione el entorno...", también Manya (s/f) observa en relación a la discapacidad, algunos esfuerzos por mejorar la calidad de vida de las personas con discapacidad; en diversos ámbitos de la vida como: hogar, escuela, comunidad así como parte de instituciones privadas y públicas, en el plano legislativo se cuenta con un régimen legal de protección, atención integral y prevención; sin embargo estos esfuerzos no son suficientes, dado a que la gran mayoría de las personas con discapacidad no acceden a estos beneficios y siguen siendo desprotegidos y excluidos.(p.5)

La calidad de vida, en relación a la dimensión de los derechos, es regular; porque el 70.4\%(197) de las PcDF nunca hace uso de las cuotas armadas por la ley. Además; referente a la percepción de la sociedad sobre la muestra de respeto, valor y consideraciones, el $59.6 \%$ de las PcDF respondieron que "a veces", percibe que la sociedad le muestra respeto valor y con- 
sideraciones. Estos resultados se sostienen en Jara (2011) cuando alude “... toda persona tiene derecho a tener trabajo y desarrollarse en éste, a pesar de que tengan algún tipo de limitación física, mental, social o cognitiva y es la sociedad quien debe brindar las condiciones para cumplir con los derechos de las personas con discapacidad" también se respalda en Hernández (2004) cuando señala que se hace necesario modificar y adaptar los entornos inmediatos, las viviendas, las edificaciones y los parques, utilizando ayudas técnicas al alcance de todos los niveles sociales, con el apoyo indispensable del Estado, desde el punto de vista legislativo, urbanístico y divulgativo, y la vinculación del sector empresarial y privado para obtener mayores y más rápidos recursos.

Los datos obtenidos, permiten afirmar que la situación laboral de las personas con discapacidad física de 18 a 65 años del OMAPED Huancayo, es predominantemente informal y la calidad de vida en todas las dimensiones son regulares.

El bienestar material es mala, aseverando que la calidad de vida que presentan las personas con discapacidad es regular con una media de 2,1624. Estos resultados se sustentan en Campen y Cardol (2009) cuando declaran que "las personas con discapacidades físicas crónicas participan menos en el trabajo remunerado y voluntario y están menos satisfechas con sus vidas que las personas sin problemas de salud.

\section{Conclusiones}

La situación laboral de las personas con discapacidad física del OMAPED Huancayo es predominantemente informal, debido al bajo nivel de instrucción como es secundaria completa e incompleta; motivo por lo que la mayoría no acceden a mejores oportunidades laborales.

La calidad de vida de las personas con discapacidad física del OMAPED Huancayo, es regular.

La situación laboral de la mayoría de las PcDF del OMAPED Huancayo, es mala porque el mayor porcentaje de trabajadores se encuentran en el sector informal por su bajo nivel de instrucción, ingresos económicos irrisorios, dificultades para trasladarse y escaso apoyo para su seguridad y protección, así mismo la calidad de vida que tienen es regular en todas sus dimensiones.

\section{Recomendaciones}

Se sugiere profundizar el estudio de la situación laboral de las personas con discapacidad física, porque además de tener una situación laboral formal e informal algunos no se ubican en ninguno de ellos, solo apoyan a sus familiares.

Continuar con el estudio desde un enfoque cualitativo, con el propósito de conocer en profundidad y con mayor detalle la situación laboral en la que se encuentran las personas con discapacidad física.

Se sugieren investigar, que programas sociales están dirigidas al apoyo de las personas con discapacidad física, por la situación económica social en que se encuentran. 
Alcover y Pérez (2011). Trabajadores con discapacidad: problemas, retos y principios de actuación en salud ocupacional. Medicina y Seguridad del Trabajo. Universidad Rey Juan Carlos. Madrid España 206- 223. http://scielo.isciii.es/pdf/mesetra/v57s1/actualizacion11.pdf

Álvarez (2014). Calidad de vida y experiencias de inserción laboral de personas con discapacidad intelectual en la administración pública de la comunidad de Foral de Navarra. Tesis de Maestría de la facultad de Ciencias Humanas y sociales del Departamento de Trabajo Social de la Universidad Pública de Navarra.

Ávila (2011). Políticas públicas y discapacidad. Investigaciones andinas 14 (24) Recuperado:

$$
\text { http://www.scielo.org.co/pdf/inan/v14n24/v14n24ao8.pdf }
$$

Bara (2015). Empleo de personas con discapacidad Rumania publicado por Elsevier Ltd. Universidad 5. Recuperado 06/09/2017.

http://www.sciencedirect.com/science/article/pii/S1877042815028001

Bolaños (2011). El derecho laboral y las personas con discapacidad en el Perú: Estándares internacionales y análisis de las implicancias de la ley general de la persona con discapacidad y su reglamento. Tesis de la Universidad de San Martín de Porres, Lima. Perú.

Brown R, Bayer M, Brown P (1992). Empoderamiento y desventajas del desarrollo: opciones y calidad de vida. Londres: Chapman \& Hall. 268-269.

Campen C. y Cardol M. (2009). Cuando el trabajo y la satisfacción con la vida no van de la mano: Barreras de salud y recursos personales en la participación de personas con discapacidad física crónica. Elsevier Recuperado en 07/o9/2017. http://www.sciencedirect. com/science/article/pii/So277953609002433

Cardona, D., Agudelo, A., Restrepo, Sánchez, L., y Segura A. (2014) Calidad de vida de las personas en situación de discapacidad física. Medellín, 2011. Revista, Ces. Salud Pública 5 (2). pp.137146. http://revistas.ces.edu.co/index.php/ces_salud_publica/article/view/3176

Cardona, D., Agudelo, A., Restrepo, L. y Segura A. (2012) Educación, salud, empleo de la población con algún tipo de limitación. Medellín, 2008. Hacia la promoción de la salud. 17(1), 77-91.

Hernández (2004) Las personas con discapacidad. Aquichan 4. (4) 60-65.

Recuperado: http://www.scielo.org.co/pdf/aqui/v4n1/v4n1ao8.pdf

Inquilla (2014) Discapacidad, Pobreza y Desigualdad Social en Puno: Un Tema Multidimensional y Multifactorial desde la perspectiva sociológica - Perú. Comunicación 6 (1).Recuperado:

\section{http://www.scielo.org.pe/pdf/comunica/v6n1/ao3v6n1.pdf}

Jara, M., (2011) Derecho al trabajo de la persona con discapacidad en Costa Rica. Artículo de la Universidad de Costa Rica. http://www.binasss.sa.cr/bibliotecas/bhp/cupula/v25n1-2/art5.pdf

Larrayoz A., (2014) Calidad de vida y experiencias de inserción laboral de personas con discapacidad intelectual en la administración pública de la Comunidad Foral de Navarra Dpto. de trabajo Social, Fac. de Ciencias Humanas y Sociales. Universidad Pública de Navarra España.

https://academica-e.unavarra.es/bitstream/handle/2454/14551/80062_Larrayoz\%2oEchart e\%2 $\mathrm{C} \% 20$ Alicia.pdf? sequence $=1 \&$ is Allowed $=y$ 
Manya A. (s/f) Discapacidad y Calidad de Vida. Universidad de San Martín de Porres. Lima Perú. http://www.revistaliberabit.com/es/revistas/liberabitog/angel_manya_aspilcueta.pdf

Maldonado, S., (2004) Trabajo y discapacidad en el Perú, mercado laboral, políticas públicas e inclusión social de las personas con discapacidad, Informe de consultoría sobre trabajo y discapacidad de la comisión de Estudios de Discapacidad (CEEDIS) del congreso de República.

Meyer, B.., \& Mok, W. (2018). Disability, earnings, income and consumption . Journal of Public Economics. University of Chicago, United States of America. https://doi.org/10.1016/j. jpubeco.2018.06.011

Morales, J., Gaviria, E. y Cuadrado, I. (2007). Psicología social (3a. ed.). Madrid: Mc Graw-Hill.

Oficina Internacional de trabajo OIT (2012) Consejo de administración Ginebra -Suiza. https:// www.ilo.org/wcmsp5/groups/public/---ed_norm/---relconf/documents/meetingdocument/ wcms_191382.pdf

OMS y el Banco Mundial (2011). Informe Mundial sobre la discapacidad. Recuperado https://www. who.int/disabilities/world_report/2011/summary_es.pdf

Schalock, R. y Verdugo, M. (2002) El concepto de calidad de vida en los servicios y apoyos para personas con discapacidad intelectual. Revista Española 38 (4) 21-36. Recuperado de:

https://www.plenainclusion.org/sites/default/files/224_articulos2.pdf

Urzúa A. y Caqueo A. (2012) Calidad de vida, una revisión teórica del concepto. Quality of life: A theoretical review. Terapia psicológica 30 (1): 61-71.

https://scielo.conicyt.cl/pdf/terpsicol/v3on1/arto6.pdf

Velarde E. y Ávila F. (2002) Evaluación de la calidad de vida. Salud pública de México 44 (4).

https://www.scielosp.org/article/spm/2002.v44n4/349-361/

Vidal, R., y Cornejo C. (2016). Trabajo y discapacidad: una mirada crítica a la inclusión al empleo. Papeles de Trabajo No 31 - Julio 2016 - ISSN 1852-4508 Centro de Estudios Interdisciplinarios en Etnolingüística y Antropología Sociocultural. 